\title{
EFFECT OF PAREDRINOL ( $\alpha-N$-DIMETHYL-p-HYDROXYPHENETHYLAMINE) ON SODIUM NITRITE COLLAPSE AND ON CLINICAL SHOCK
}

\author{
By PAUL KUNKEL, EUGENE A. STEAD, JR., AND SOMA WEISS \\ (From the Thorndike Memorial Laboratory, Second and Fourth Medical Services (Harvard), \\ Boston City Hospital, and the Department of Medicine, Harvard Medical School, Boston)
}

(Received for publication June 19, 1939)

Previous studies (1) (2) have demonstrated that circulatory collapse similar to that often observed in disease and suitable for experimental study is induced in normal subjects in the upright position by administering sodium nitrite. This collapse results from decreased venous tone, which causes pooling of blood in the veins and venules of the dependent portions of the body. Consequently, the cardiac output decreases and, with the ensuing cerebral anoxia, syncope usually occurs. Epinephrin and pitressin have been found to be ineffective in preventing this type of collapse and syncope (3) (4). Indeed, in a number of cases pitressin aggravated the circulatory collapse. Epinephrin causes a definite increase in venous tone (3), but at the same time it necessitates a greater cardiac output because of the increased metabolic rate and widespread chemical changes in certain tissues. Thus, while in sodium nitrite collapse epinephrin may increase the venous return to the heart to some extent, it does not cause enough increase to compensate for the augmented tissue requirements. Pitressin does not affect the venous tone and therefore has no favorable action in the type of collapse investigated. In addition, both these drugs produce arteriolar constriction in certain organs (3) (4). This action is unfavorable for, as venous pooling occurs, compensatory reflex arteriolar constriction takes place. An increase in this arteriolar constriction by drugs, therefore, further reduces the tissue blood flow and accentuates tissue anoxia.

Since in sodium nitrite collapse venous pooling and reflex arteriolar constriction occur, the ideal drug for the prevention of such collapse should produce constriction of the veins and venous reservoirs without arteriolar constriction, or possibly with arteriolar dilatation. Such a substance should not cause increased oxygen consumption or widespread chemical changes in the body. From studies of normal subjects it was found that paredrinol ${ }^{1}$ ( $\alpha$-N-dimethyl-p-hydroxyphenethylamine) fulfilled several of these requirements (5). Therefore its action was investigated in the collapse induced by sodium nitrite and in that accompanying postural hypotension and acute infectious disease.

\section{METHODS}

In 7 subjects with normal cardiovascular systems collapse ending in syncope was induced by the administration of sodium nitrite. The heart rate was counted by arterial palpation or by precordial auscultation. Arterial pressure was measured in the arm at heart level by the auscultatory method, using a mercury manometer. The blood flow in the hand was determined by Freeman's modification (6) of the plethysmographic method of Hewlett and Van Zwaluwenburg (7), and the venous tone by the plethysmographic method of Capps (8). The subject rested on a tilting table in the horizontal position for at least 30 minutes after all apparatus had been adjusted and the water in the plethysmograph had reached the desired temperature.

The following experiments were carried out in each of the 7 subjects on different days: (1) The oral administration of 0.18 gram ( 3 grains) of sodium nitrite was followed in 15 minutes by the intramuscular injection of sterile saline and in 25 minutes by tilting the subject to an angle of 75 degrees above the horizontal. When collapse and syncope developed the subject was immediately returned to the horizontal position. (2) The oral administration of 0.18 gram ( 3 grains) of sodium nitrite was followed in 15 minutes by the intramuscular injection of from 25 to $45 \mathrm{mgm}$. of paredrinol and in $25 \mathrm{~min}-$ utes by tilting to the upright position (75 degrees). Unless collapse ending in syncope occurred the subjects were allowed to stand motionless for 30 minutes and then returned to the horizontal position. If the subject did not develop collapse when paredrinol was used, the experiment was repeated with sodium nitrite followed by the injection of sterile saline in order to ascertain that the person was still sensitive to nitrite. The order of the experiments was varied and the subjects were not told what medication they were receiving. In addition to these experiments on normal subjects, the effect of paredrinol on the arterial pressure, the heart rate and the

1 The paredrinol sulphate used in this investigation was supplied through the courtesy of the Smith, Kline \& French Laboratories, Philadelphia. 
clinical state of a group of patients in medical shock was studied.

\section{RESULTS}

Sodium nitrite collapse. In 4 subjects paredrinol prevented the development of circulatory collapse ending in syncope, which had previously followed the oral administration of 0.18 gram ( 3 grains) of sodium nitrite and tilting to the upright position. With each of these 4 subjects 2 experiments in which sterile saline was substituted for paredrinol had resulted in collapse ending in syncope. Figure 1 shows the effect in a normal subject of the oral administration of sodium nitrite followed by tilting to the upright position. The heart rate increased, the arterial pressure, particularly the systolic pressure, fell progressively and the pulse pressure became very small. The blood flow in the hand at $43^{\circ} \mathrm{C}$. decreased due to reflex arteriolar constriction and reduced cardiac output. Subsequently the subject became pale, began to yawn and sweat, complained of nausea and dizzi- ness and finally lost consciousness. When the subject was returned to the horizontal position all symptoms rapidly disappeared. The blood pressure, heart rate and blood flow in the hand quickly returned to normal.

The effect in the same subject of the intramuscular injection of $25 \mathrm{mgm}$. of paredrinol 15 minutes after the administration of the sodium nitrite and 10 minutes before tilting is shown in Figure 2. As previously shown (5), sodium nitrite has little effect on the development of paredrinol hypertension in the horizontal position. This subject, however, was tilted before the paredrinol hypertension had fully developed. The effect of the upright position on the circulation was immediately obvious. The arterial pressure, particularly the diastolic, fell, the heart rate increased greatly and the blood flow in the hand at $43^{\circ} \mathrm{C}$. decreased. The subject nevertheless stood for 30 minutes without difficulty, at the end of which time he was returned to the horizontal posi-

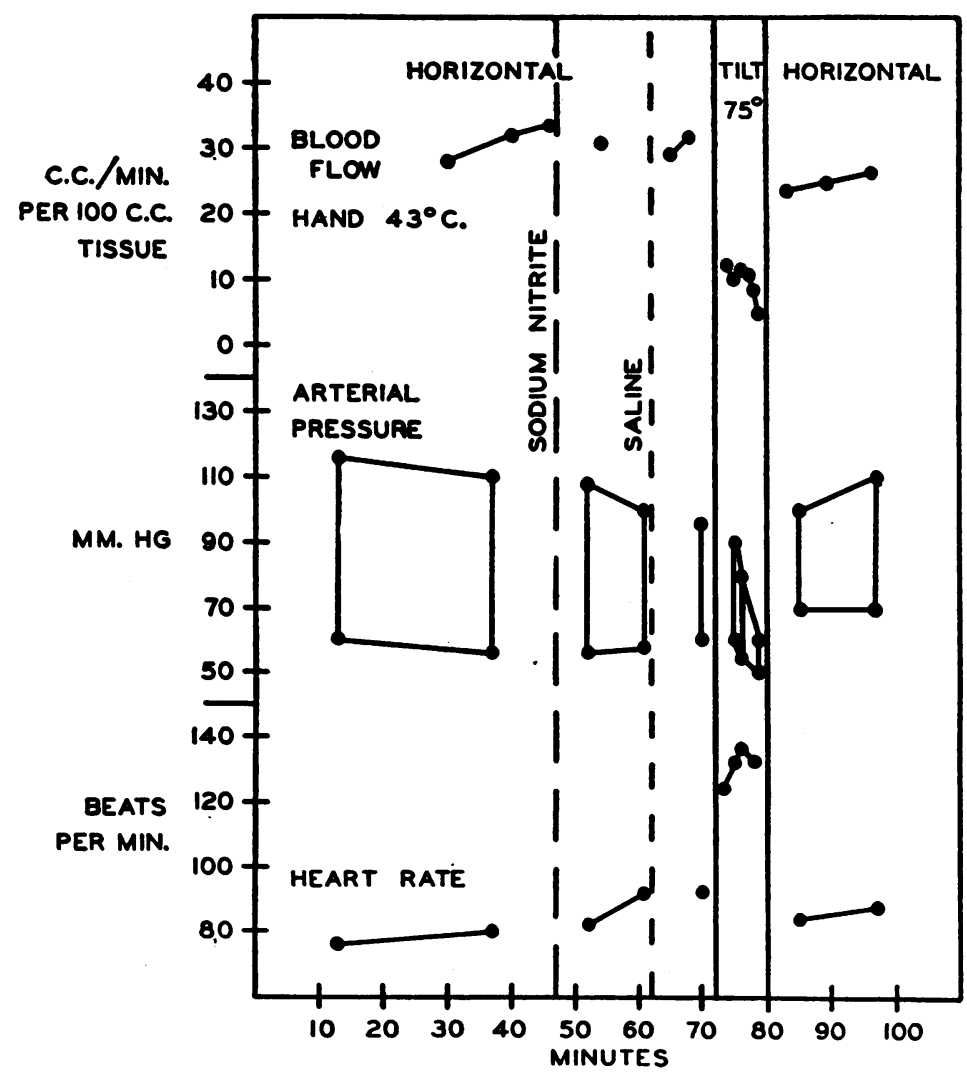

Fig. 1. Collapse Induced by Sodium Nitrite in a Normal Male 


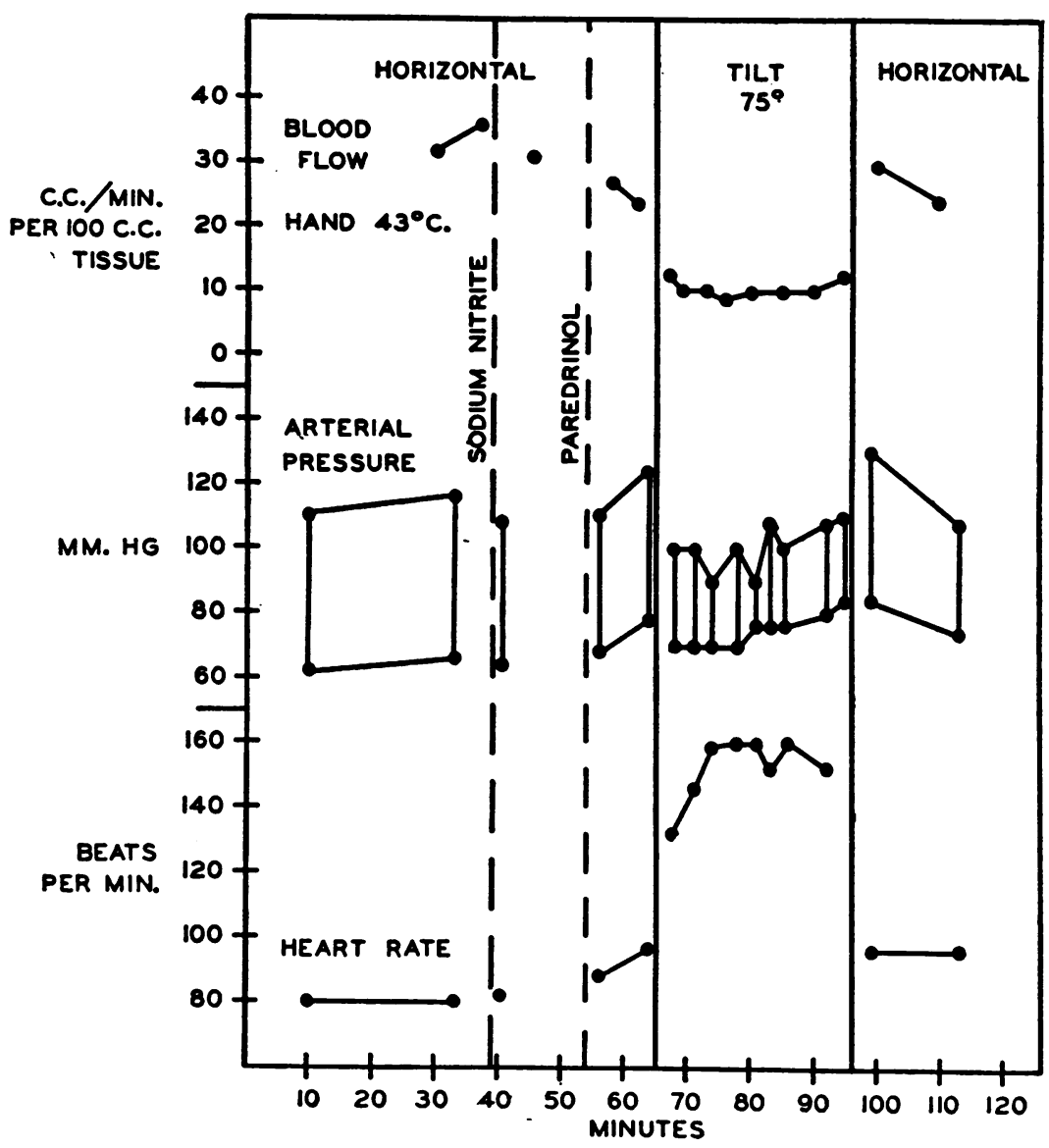

Fig. 2. The Prevention of the Collapse Induced by Sodium Nitrite by Means of the Intramuscular Injection of 25 mgm. of Paredrinol

The same subject as in Figure 1.

tion. The experiments in 3 other subjects were similar.

In 3 subjects paredrinol had no effect on the course of the collapse induced by sodium nitrite in the upright position. There was no correlation between the degree of hypertension produced by paredrinol in subjects in the horizontal position and the ability of the subject to stand after nitrite. In 2 of these subjects, in whom paredrinol failed to prevent collapse, the arterial pressure before tilting had risen from normal to $174 \mathrm{~mm}$. and 180 $\mathrm{mm}$. $\mathrm{Hg}$ systolic, and to $74 \mathrm{~mm}$. and $80 \mathrm{~mm}$. $\mathrm{Hg}$ diastolic, respectively. When the subject was placed in the upright position, however, the arterial pressure quickly fell to levels indicative of collapse, and syncope occurred. Likewise there was no close correlation between the length of time that the subject could stand when only sodium nitrite was given and the effectiveness of paredrinol in preventing collapse. Thus 1 subject stood for only 5 minutes after the administration of sodium nitrite, but he stood for 30 minutes without difficulty when the ingestion of nitrite was followed by the injection of paredrinol. In a second subject who stood for 10 minutes when only sodium nitrite was given, paredrinol was totally ineffective in preventing collapse.

The circulatory collapse induced by sodium nitrite in the upright position closely resembles that seen clinically in subjects with postural hypotension. In both conditions cerebral anoxia is produced by pooling of the blood in the venous side of the circulatory system. Paredrinol was therefore given to 2 subjects with severe postural hypotension in whom attacks of syncope normally occurred after 6 to 10 minutes of motionless 
standing. After the intramuscular injection of from 20 to $35 \mathrm{mgm}$. of paredrinol, these subjects stood for 30 minutes without developing any symptoms of collapse.

Clinical shock. Paredrinol was given either intramuscularly or intravenously in doses of from 15 to $50 \mathrm{mgm}$. to 10 patients in severe medical shock. Eight had acute infectious diseases; 2 had miliary tuberculosis. In addition to a low arterial pressure and a small pulse pressure, the majority of the patients had cold extremities, which indicated a slow peripheral blood flow. In 7 of the 10 patients a rise in arterial pressure was produced; in 3 no rise occurred. In only 2 of the patients did the arterial pressure rise above the normal level. The response of these cases in clinical collapse to paredrinol differed from that in normal subjects in at least 3 ways: (1) Much larger doses of the drug (up to 50 mgm. intravenously) were required to produce a rise in arterial pressure, and the effect of the drug lasted only from 15 to 30 minutes, while in the normal subject the effect of an intramuscular injection of $25 \mathrm{mgm}$. of paredrinol usually lasted 60 minutes; (2) the heart rate was as a rule greatly increased, while in normal subjects it was usually decreased; (3) repeated injections of the same amount of the drug a short time after the arterial pressure had fallen to the original level produced less response each time, until in most cases large doses no longer had any effect on the arterial pressure. In 3 of these subjects in shock the blood flow in the dilated hand was measured. In 2 of these cases in which paredrinol did not produce a rise in arterial pressure, the blood flow in the hand decreased and the extremities became cold; in one in which paredrinol produced a definite rise in blood pressure the blood flow in the hand did not definitely increase. In the third subject the usual spontaneous fluctuations in vasomotor tone and the reflex vasoconstrictor responses to sensory stimuli were absent before the administration of paredrinol because of the presence of shock. Paredrinol did not restore these functions although it raised the blood pressure.

Clinical improvement was definitely established by the administration of paredrinol in only 2 of the 10 cases with circulatory collapse. In one of these patients collapse followed the adminis- tration of horse serum. The second subject, who had an acute streptococcal infection of the throat, collapsed when she was placed in Fowler's (semirecumbent) position. After this patient became afebrile, her postural reactions were investigated. When she was tilted to an angle of 75 degrees syncope developed in 3 minutes, whereupon she was returned to the horizontal position. She was then given $25 \mathrm{mgm}$. of paredrinol intramuscularly and the arterial pressure rose from $100 \mathrm{~mm}$. systolic and $60 \mathrm{~mm}$. diastolic to $160 \mathrm{~mm}$. systolic and 80 $\mathrm{mm}$. diastolic. The heart rate dropped from 70 to 63 beats per minute. On being tilted to an angle of 75 degrees the patient became paler but stood without difficulty for 15 minutes, when she developed nausea and was returned to the horizontal position. At that time the blood flow in the hand was fairly well maintained. The arterial pressure was $100 \mathrm{~mm}$. systolic and $70 \mathrm{~mm}$. diastolic; the heart rate was 140 . Thus in this patient both spontaneous collapse in the course of a streptococcus infection and subsequently induced collapse and syncope were improved by paredrinol.

\section{DISCUSSION}

The effects in normal subjects of the intramuscular administration of paredrinol have been reported (5). The subjects experienced no symptoms except palpitation. The apex impulse became more forceful, arterial pulsations in the neck more prominent and the heart sounds increased in intensity. The intramuscular injection of $25 \mathrm{mgm}$. of the drug produced in 10 normal subjects an average arterial pressure of $173 \mathrm{~mm}$. systolic and $92 \mathrm{~mm}$. diastolic. The pulse rate usually fell. The venous pressure was elevated by from 30 to $40 \mathrm{~mm}$. of water. The venous tone was also increased. The blood flow in the hand at $43^{\circ} \mathrm{C}$. became slower, but that in the foot, forearm and calf was unchanged. The cardiac output, circulation time and metabolic rate showed no significant changes.

The favorable effect of paredrinol on the collapse induced by sodium nitrite and posture results mainly from the action of the drug on the venous system. Experiments on animals have shown that paredrinol causes an increase in venous tone and an emptying out of the venous reservoirs. Studies on normal subjects have 
demonstrated that the venous tone is increased and that the venous pressure rises by from 30 to $40 \mathrm{~mm}$. of water. Thus the action of the drug on the veins (Figure 3 ) is antagonistic to that of sodium nitrite, which has been shown to decrease the venous tone and to produce hypotension and collapse in the upright position by venous pooling in the dependent portions of the body. Fortunately, paredrinol has no unfavorable side reactions. It does not produce pain. It does not increase metabolism, as does epinephrin, and thus create a need for greater cardiac output. It does not cause marked arteriolar constriction.

Epinephrin (3) has likewise been shown to increase the venous tone (Figure 3). This effect, which should be useful in collapse caused by venous pooling, is, however, overbalanced by the rise in metabolism and by chemical changes initiated in the muscles and liver which probably create a need for a greater cardiac output. Pitressin does not increase the venous tone (Figure 3 ), and therefore is not useful in this type of collapse. In addition, both epinephrin and pitressin cause arteriolar constriction in certain organs. This is an unfavorable action because the arterioles have already undergone reflex contraction due to the fall in blood pressure, and the additional arteriolar constriction induced by the drug reduces the already diminished blood supply to the tissue.

After the administration of sodium nitrite and paredrinol the heart rate always becomes very rapid when the subject is tilted to the upright position. The slow heart rate usually present in normal subjects after the injection of paredrinol results from vagal inhibition from stimuli passing through the carotid sinus and aortic nerves. The heart rate becomes rapid, therefore, if the vagal influence is eliminated by atropine (8), or if the stimulus to the carotid sinus and aortic nerve endings is removed by a fall in blood pressure, as is the case in nitrite collapse. Similar changes are present in clinical collapse. In these cases the heart rate usually increases when paredrinol is given, probably because the pressure does not rise high enough to produce vagal inhibition by stimulation of the sensitive vascular areas. Experiments with atropine have shown that paredrinol causes an increase in heart rate when vagal influence is eliminated.

In 7 of the 10 patients in severe clinical collapse, the injection of paredrinol resulted in a rise

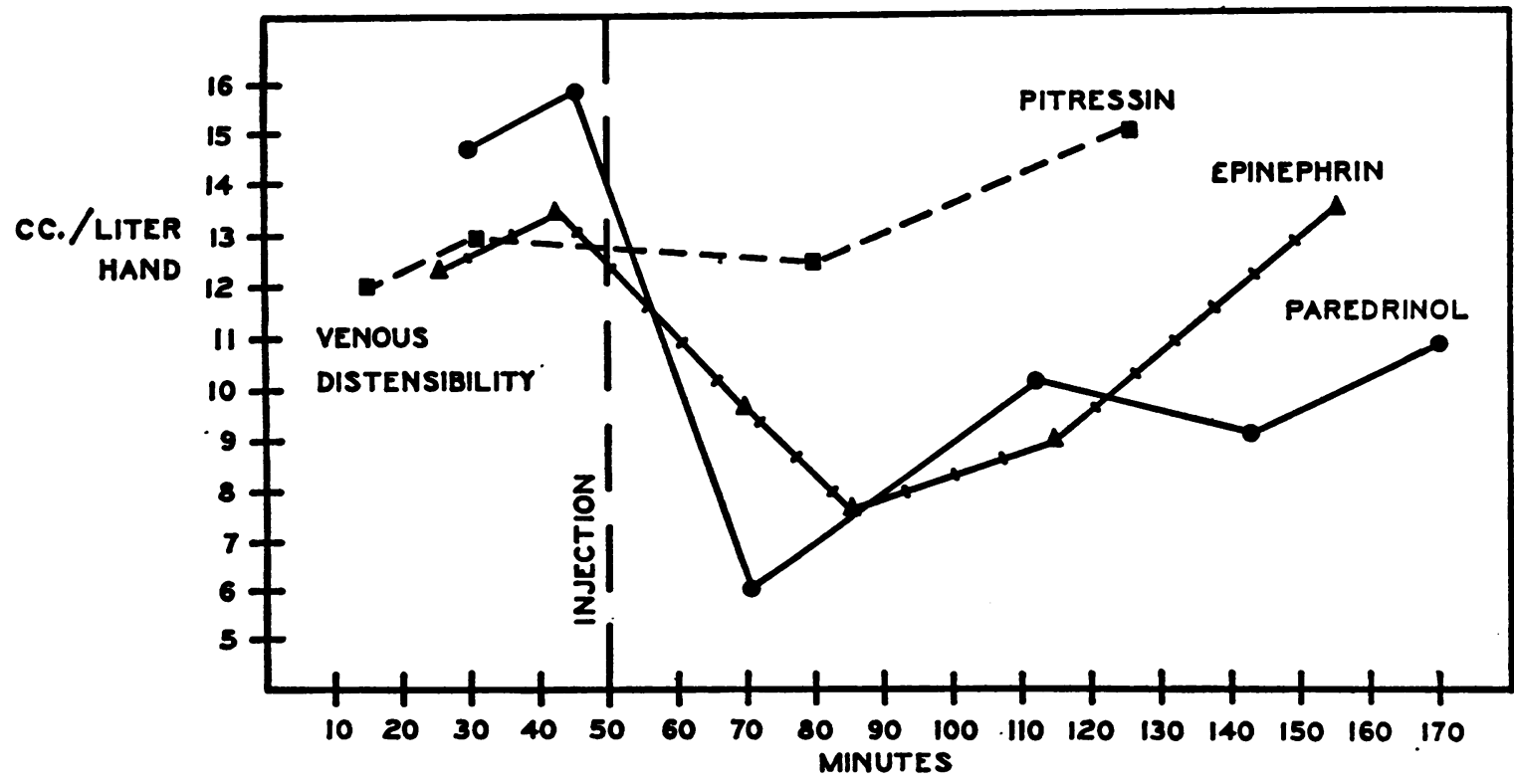

Fig. 3. The Effect of the Injection of Paredrinol, Epinephrin and Pitressin on the Venous Tone in the Sympathectomized (Preganglionic) Hand

An occluding pressure of $30 \mathrm{~mm}$. was applied to the wrist and the resulting increase in hand volume was measured. A decrease in venous distensibility indicates an increase in venous tone. 
of the arterial pressure. With the exception of 2 cases, however, these patients showed no clinical improvement. The height of the arterial pressure has been demonstrated to be an unsatisfactory guide to the general condition of a patient in collapse (3). Additional studies will be necessary, therefore, in order to ascertain whether such cases actually benefit by the observed rise in blood pressure. In 2 of the cases in which the arterial pressure did not rise, paredrinol caused a decrease in blood flow in the hand and appeared to increase the severity of the collapse. The evidence available indicates that paredrinol raises the arterial pressure, at least in part, by mobilizing blood from the venous reservoirs and by increasing the venous tone. It is possible that the amount of blood present in the minute vessels (blood reservoirs) is the factor which determines whether paredrinol will be effective. Hence it may not be helpful, and indeed may be harmful, in the types of collapse which result primarily from the loss of fluid from the blood stream rather than from the pooling of blood in a dilated venous system.

In the cases of medical shock studied, the decreasing effectiveness of successive doses of paredrinol given at short intervals is very striking. This is not the case in normal subjects, in whom the arterial pressure can be maintained at hypertensive levels for several hours without difficulty by repeated doses of the drug. Rein (9) states that in dogs successive doses of paredrinol produce the same cardiovascular responses as a single injection. Eichler (10) concludes that in cats paredrinol becomes less effective with repeated administration. The decrease in the effectiveness of the drug on repeated administration in these cases of clinical collapse may be due to the fact that the vascular storage depots of the blood are already depleted, and that the first dose of the drug completely empties them.

\section{SUMMARY AND CONCLUSIONS}

1. From theoretical considerations paredrinol ( $\alpha$-N-dimethyl-p-hydroxyphenethylamine) should be effective in preventing the sodium nitrite collapse induced in the upright position because paredrinol increases the venous tone without at the same time increasing the metabolism of the tissues or causing marked arteriolar constriction.
2. In 4 normal subjects the intramuscular injection of $25 \mathrm{mgm}$. of paredrinol prevented the collapse induced in the upright position by sodium nitrite. In 3 others it had no effect.

3 . In 2 subjects with severe postural hypotension, symptoms of cerebral anoxia were prevented by the use of paredrinol.

4. In 7 of the 10 cases in severe clinical shock resulting from infectious disease, the intramuscular or intravenous injection of from 15 to 50 $\mathrm{mgm}$. of paredrinol caused a rise in arterial pressure. Only 2 of these cases showed definite clinical improvement.

5. In 1 patient paredrinol was effective both in the collapse induced by an acute streptococcal pharyngitis and in the collapse subsequently induced by motionless standing.

6. The responses of the patients in severe clinical collapse differed from those in the normal subject as follows: (a) From 2 to 4 times the amount of paredrinol was required to cause a significant elevation of blood pressure, and even then the arterial pressure rarely increased to hypertensive levels; $(b)$ the heart rate was usually increased instead of decreased; $(c)$ repeated doses of the drug, when given after the blood pressure had returned to normal, failed to be as effective as the original injection.

7. Paredrinol is a useful drug in the treatment of collapse caused by the pooling of blood within a dilated venous system. This study suggests, however, that in shock resulting primarily from loss of fluid from the blood stream the drug may not be helpful, or it may even be harmful.

This investigation was carried out with the technical assistance of Miss Sophia M. Simmons, S.B.

\section{BIBLIOGRAPHY}

1. Weiss, S., Wilkins, R. W., and Haynes, F. W., The nature of circulatory collapse induced by sodium nitrite. J. Clin. Invest., 1937, 16, 73.

2. Wilkins, R. W., Haynes, F. W., and Weiss, S., The rôle of the venous system in circulatory collapse induced by sodium nitrite. J. Clin. Invest., 1937, $16,85$.

3. Wilkins, R. W., Weiss, S., and Haynes, F. W., The effect of epinephrin in circulatory collapse induced by sodium nitrite. J. Clin. Invest., 1938, 17, 41.

4. Stead, E. A., Jr., Kunkel, P., and Weiss, S., Effect of pitressin in circulatory collapse induced by sodium nitrite. J. Clin. Invest., 1939, 18, 673. 
5. Stead, E. A., Jr., and Kunkel, P., Mechanism of the arterial hypertension induced by paredrinol $(\alpha-\mathrm{N}$ dimethyl-p-hydroxyphenethylamine). J. Clin. Invest., 1939, 18, 439.

6. Freeman, N. E., The effect of temperature on the rate of blood flow in the normal and in the sympathectomized hand. Am. J. Physiol., 1935, 113, 384.

7. Hewlett, A. W., and Van Zwaluwenburg, J. G., The rate of blood flow in the arm. Heart, 1909-10, 1, 87.

8. Capps, R. B., A method for measuring tone and re- flex constriction of the capillaries, venules and veins of the human hand with the results in normal and diseased states. J. Clin. Invest., 1936, 15, 229.

9. Rein, H., Utber die Kreislauf- und Stoffwechselwirkungen des $\beta$-(p-Oxyphenyl)-Isopropyl-Methylamins. Arch. f. exper. Path. u. Pharmakol., 1937, 187, 617.

10. Eichler, O., Zur Pharmakologie des Veritols (H75) und zu seiner Einordnung in die Reihe der bekannten Adrenalinkörper. Arch. f. exper. Path. u. Pharmakol., 1937, 187, 429. 